\title{
Glycosylation of tyrosinase is a determinant of melanin production in cultured melanoma cells
}

\author{
MARI MIKAMI $^{1}$, TOMONORI SONOKI $^{1}$, MINASE ITO $^{1}$, YOKO FUNASAKA $^{2}$, \\ TAMIO SUZUKI ${ }^{3}$ and YOHTARO KATAGATA ${ }^{1}$ \\ ${ }^{1}$ Faculty of Agriculture and Life Sciences, Hirosaki University, Hirosaki, Aomori 036-8561; ${ }^{2}$ Nippon Medical School, \\ Bunkyo, Tokyo 113-8602; ${ }^{3}$ Faculty of Medicine, Yamagata University, Yamagata 990-9585, Japan
}

Received February 20, 2013; Accepted July 9, 2013

DOI: $10.3892 / \mathrm{mmr} .2013 .1602$

\begin{abstract}
The majority of malignant melanoma cell types are able to produce melanin and the degree of melanin synthesis in various types of cultured cell line differs. In this study, we evaluated three types of cultured cell line, MNT-1, HM3KO and G-361, with differing melanin production levels. The level was greatest in the MNT-1 cells, lower in the HM3KO cells and lowest in the G-361 cells. In addition, a positive correlation between melanin production and tyrosinase activity was observed. The molecular masses of tyrosinases from $\mathrm{HM} 3 \mathrm{KO}$ and G-361 cells were marginally lower than those from MNT-1 cells. Glycosylation inhibitor treatment on MNT-1 cells caused decreases in the molecular mass of tyrosinase, its activity and melanin production. An immunoprecipitation assay using anti-tyrosinase indicated that the immature glycosylated tyrosinases were associated with a type of chaperone, Hsp70. The interaction between tyrosinase and Hsp70 was also detected in HM3KO and G-361 cells. The results indicated that the immature glycosylation of tyrosinase has a critical effect on the melanin-producing ability of melanoma cells.
\end{abstract}

\section{Introduction}

Melanin is important in protecting the skin from the harmful effects of ultraviolet irradiation and absorbing toxic drugs and chemicals. Melanin is synthesized in two predominant forms, black-brown pigments (eumelanin) and red-yellow pigments (pheomelanin), within the melanosomes of melanocytes by at least three melanogenic enzymes. Tyrosinase (EC1.14.18.1) is one of the predominant enzymes mediated by the melanin production process in melanosomes (1). The enzyme catalyzes the rate-limiting step of the hydroxylation of tyrosine to dihydroxyphenylalanine (DOPA) and its further oxidation

Correspondence to: Dr Tomonori Sonoki, Faculty of Agriculture and Life Sciences, Hirosaki University, 3 Bunkyo-cho, Hirosaki, Aomori 036-8561, Japan

E-mail: sonoki@cc.hirosaki-u.ac.jp

Key words: melanoma, melanogenesis, tyrosinase, glycosylation to DOPA-quinone (2). Melanin synthesis is maintained by a number of regulatory processes that act at various steps during the synthesis of the protein in the endoplasmic reticulum (ER), Golgi body and melanosomes, and disorders in any of these processes lead to abnormal pigmentation.

Malignant melanoma (MM) cells are derived from epidermal melanocytes and nevi (3), and numerous cultured melanoma cell lines have been established from human and mouse MMs. The majority of MMs produce melanin, and the degree of melanin synthesis differs for each type of cultured cell line (4-6). For example, MNT-1 cells were demonstrated to be highly pigmented MMs (4); however, SK-Mel-28 cells were not able to produce melanin due to a mutation in the endocytic pathway that affected the endosomal trafficking of tyrosinase (5). In addition, B16F10 cells were pigmented; whereas amelanotic melanoma was not pigmented, due to the increased expression of proteasome subunit p27 (6).

In this study, three cultured cell lines, MNT-1, HM3KO and G-361, were observed. These cell lines differed in their degrees of melanin synthesis, and thus, the determinants of the degree of melanin synthesis were investigated.

\section{Materials and methods}

Cell culture. The MNT-1 (from Dr VJ Hearing, Laboratory of Cell Biology, National Cancer Institute, National Institutes of Health, Bethesda, MD, USA), HM3KO (7,8) and G-361 (9) (supplied by the Health Science Research Resources Bank, Osaka, Japan) cell lines were cultured in Dulbecco's modified Eagle's medium (Life Technologies, Carlsbad, CA, USA) or McCoy's 5A medium (Sigma-Aldrich, St. Louis, MO, USA) containing $10 \%$ fetal bovine serum, $100 \mathrm{U} / \mathrm{ml}$ penicillin, $50 \mu \mathrm{g} / \mathrm{ml}$ streptomycin, $50 \mu \mathrm{g} / \mathrm{ml}$ kanamycin and $2.5 \mu \mathrm{g} / \mathrm{ml}$ amphotericin B (10). The study was approved by the Ethics Committee of Hirosaki University, Hirosaki, Japan

Measurement of melanin production and tyrosinase activity. Melanin production in each melanoma cell line was measured according to the method proposed by Ando et al (11) with certain modifications. Approximately $10^{6}$ cells were collected by centrifugation at $8,000 \times \mathrm{g}$ for $5 \mathrm{~min}$ and washed twice with phosphate-buffered saline (PBS), and then centrifuged at $2,000 \mathrm{xg}$ for $5 \mathrm{~min}$. The precipitate was then solubilized by 
treatment with $1 \mathrm{ml} 2 \mathrm{M} \mathrm{NaOH}$ in $10 \%$ dimethyl sulfoxide for $30 \mathrm{~min}$ at $80^{\circ} \mathrm{C}$ in a capped test tube. The absorbance of melanin in the supernatant was measured at $450 \mathrm{~nm}$. Tyrosinase activity was measured as the DOPA oxidase activity (12) with certain modifications. Approximately $10^{7}$ cells were collected by centrifugation and washed twice with PBS. Following centrifugation, the supernatant was decanted. Cells were suspended in ice-cold 0.1 M PBS containing 1\% Triton X-100 and $0.5 \mathrm{mM}$ phenylmethylsulfonyl fluoride (PMSF), and homogenized. The suspension was centrifuged for $5 \mathrm{~min}$ at $4^{\circ} \mathrm{C}$ and $1,200 \mathrm{x} \mathrm{g}$, and the supernatant was collected. Tyrosinase activity was analyzed spectrophotometrically by following the oxidation of DOPA to dopachrome at $475 \mathrm{~nm}$. Supernatant $(90 \mu \mathrm{l})$ containing $100 \mu \mathrm{g}$ crude protein was applied to a 96-well microplate and $10 \mu \mathrm{l}$ $0.1 \mathrm{M}$ L-DOPA was added. Following incubation for $1 \mathrm{~h}$ at $37^{\circ} \mathrm{C}$, the absorbance was measured.

Western blot analysis. Each cultured cell line was suspended in extraction buffer consisting of $50 \mathrm{mM}$ Tris- $\mathrm{HCl}(\mathrm{pH} 7.5)$, $150 \mathrm{mM} \mathrm{NaCl}, 1 \mathrm{mM}$ EDTA, $1 \%$ Triton X-100 and protease inhibitors $(2 \mathrm{mM}$ N-ethylmaleimide, $50 \mathrm{mg} / \mathrm{ml}$ aprotinin, $50 \mathrm{mg} / \mathrm{ml}$ leupeptin and $50 \mathrm{mg} / \mathrm{ml}$ pepstatin), and homogenized. Following centrifugation, the soluble protein in the crude extract was quantified according to the method described by Asryants (13). Proteins were separated by sodium dodecyl sulfate-polyacrylamide gel electrophoresis using $10 \%$ gels, and blotted onto polyvinylidene fluoride membranes. Primary antibodies, anti-tyrosinase and anti-Hsp70 (Santa Cruz Biotechnology, Inc., Dallas, TX, USA), as well as anti-actin (Thermo Fisher Scientific, Waltham, MA, USA), were diluted at 1:1000 in $0.5 \%$ bovine serum albumin/Tris-buffered saline and Tween 20, respectively. The horseradish-peroxidase-conjugated secondary antibody (Santa Cruz Biotechnology, Inc.) was used and antigen-antibody complexes were detected by the chemiluminescence method, using an enhanced chemiluminescence kit (GE Healthcare UK, Ltd., Buckinghamshire, UK) and X-ray film (FujiFilm Corp., Ltd., Tokyo, Japan).

Treatment of melanoma by glycosylation inhibitors, 1-deoxynojirimycin (DNJ) and D-mannojirimycin (DMJ). A total of $10^{6}$ cells of MM were incubated as described previously. After 24 h, DNJ and DMJ (14) (Enzo Life Sciences, Inc., Farmingdale, NY, USA) were added to the culture at final concentrations of $1 \mathrm{mM}$ and incubated for an additional 4 days. The treated cells were harvested and the cell-free extracts were prepared for analysis.

Immunoprecipitation with anti-tyrosinase antibody. A total of $100 \mathrm{mg}$ cells of each cultured line was suspended in $1 \mathrm{ml}$ TNE buffer and homogenized for $30 \mathrm{sec}$ at $4^{\circ} \mathrm{C}$. Protein-A Sepharose 4 Fast Flow (20 $\mu \mathrm{l}$; GE Healthcare UK, Ltd.) was added to $200 \mu \mathrm{l}$ supernatant and the mixture was mixed briefly for $1 \mathrm{~h}$ at $4^{\circ} \mathrm{C}$. The suspension was centrifuged at $19,000 \mathrm{x} g$ for $10 \mathrm{~min}$ and the supernatant was incubated with $10 \mu \mathrm{l}$ anti-tyrosinase at $4^{\circ} \mathrm{C}$ for $\sim 20 \mathrm{~h}$. Protein-A Sepharose 4 Fast Flow $(20 \mu \mathrm{l})$ was added to the mixture and mixed briefly at $4^{\circ} \mathrm{C}$ for $2 \mathrm{~h}$. After $2 \mathrm{~h}$, the mixture was centrifuged at $14,000 \mathrm{rpm}$ for $30 \mathrm{sec}$ and the precipitate was collected. The precipitate was washed five times with TNE buffer [10 mM Tris- $\mathrm{HCl}$ (pH 7.8), $0.15 \mathrm{M} \mathrm{NaCl}, 1$ mM EDTA, $1 \%$ NP-40
A

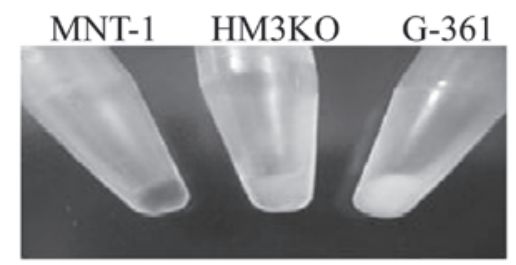

B

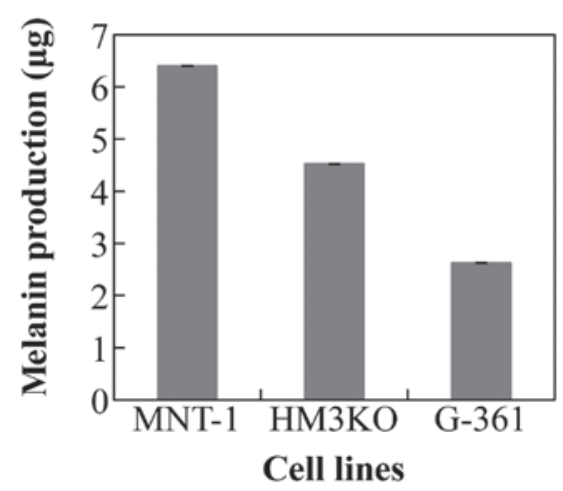

C
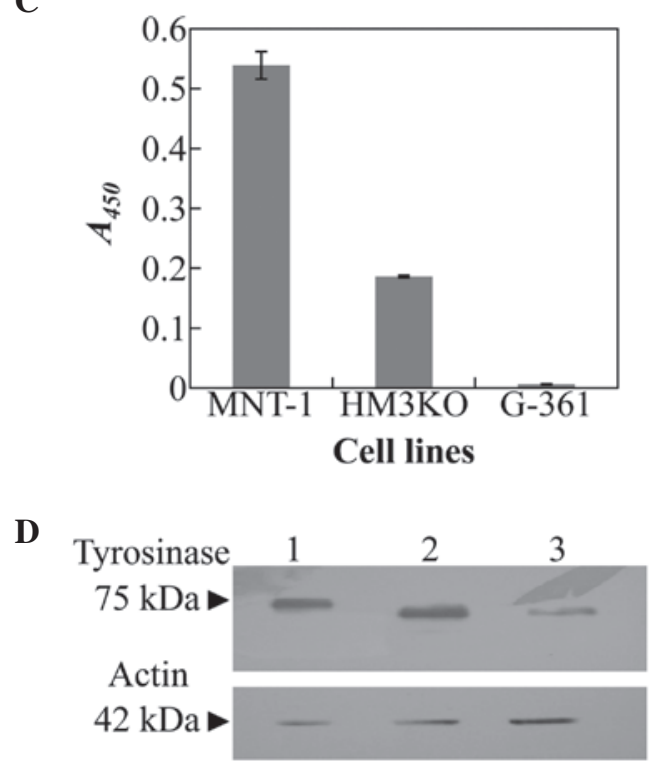

Figure 1. Characteristics of the melanin production of three types of cultured melanoma cell lines, MNT-1, HM3KO and G-361. (A) External appearance, (B) melanin production and (C) tyrosinase activity of MNT-1, HM3KO and G-361 cells. (D) Western blot analysis of expressed tyrosinase and actin (as an internal control). Lane $1,15-\mu \mathrm{g}$ extract from MNT-1 cells; lane 2, 30- $\mu \mathrm{g}$ extract from HM3KO cells; lane 3, 60- $\mu$ g extract from G-361 cells.

and $1 \mathrm{mM}$ PMSF] and the resulting precipitate was subjected to western blot analysis with Hsp70 antibody. To compare the quantity of Hsp70 associated with anti-tyrosinase among the three cultured cell lines, the signal intensity was analyzed using Scion image software (Scion Corp., National Institutes of Health, Frederick, MD, USA) and the ratios of each numerical value measured in the sample with the immunoprecipitation treatment to that without the treatment was determined.

\section{Results}

Melanin production and tyrosinase activity in the three melanoma cell lines. The external appearance of each harvested cell line is shown in Fig. 1A. The melanin production and 
A

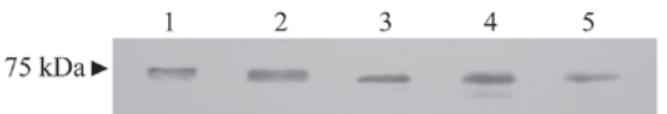

B

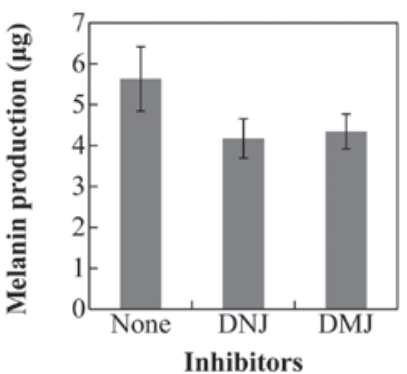

C

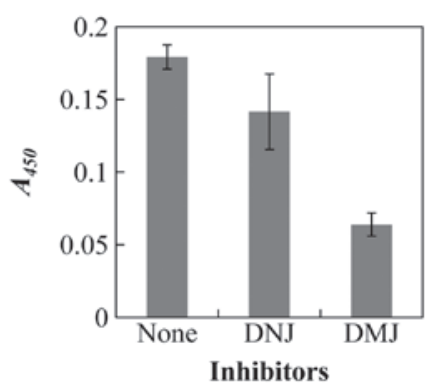

Figure 2. Affect of the glycosylation inhibitors on the tyrosinase and melanin production in MNT-1 cells. (A) Western blot analysis of the expressed tyrosinase in each cell line. Lane 1, 15- $\mu \mathrm{g}$ extract from MNT-1 cells; lane 2, 15- $\mu \mathrm{g}$ extract from 1-deoxynojirimycin (DNJ)-treated MNT-1 cells; lane 3, 15- $\mu \mathrm{g}$ extract from D-mannojirimycin (DMJ)-treated MNT-1 cells; lane 4, 30- $\mu \mathrm{g}$ extract from HM3KO cells; lane 5, 60- $\mu \mathrm{g}$ extract from G-361 cells. (B) Melanin production in MNT-1 cells with or without the inhibitor treatments. (C) Tyrosinase activity in MNT-1 cells with or without the inhibitor treatments.
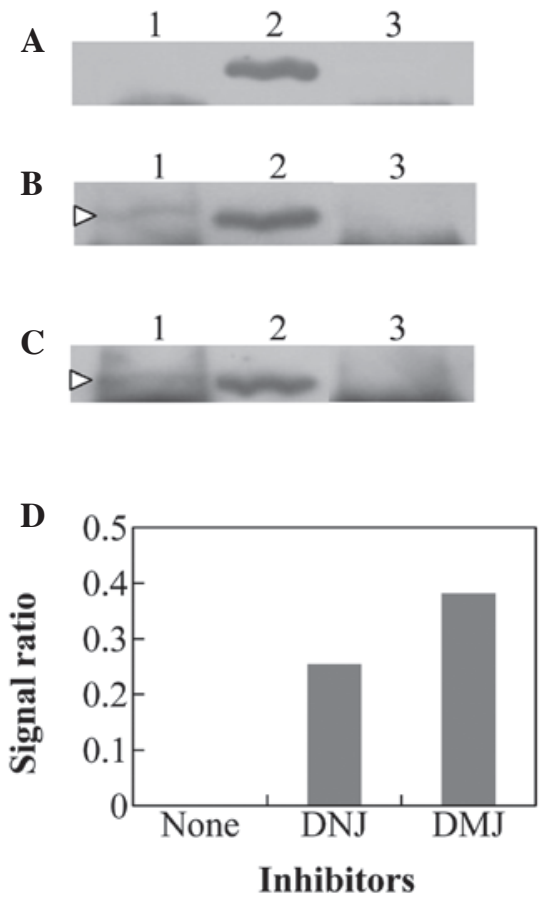

Figure 3. Immunoprecipitation of Hsp70 with anti-tyrosinase in MNT-1. Extracts were prepared from (A) MNT-1 cells, (B) 1-deoxynojirimycin (DNJ)-treated MNT-1 cells and (C) D-mannojirimycin (DMJ)-treated MNT-1 cells. Each extract was immunoprecipitated with anti-tyrosinase antibody. The immunoprecipitated products and extracts were analyzed by western blot analysis with anti-Hsp70 antibody. Lane 1, immunoprecipitated fraction with anti-tyrosinase; lane 2, extract without the immunoprecipitation treatment; lane 3, nonimmune IgG. The immuoprecipitated Hsp70 with antityrosinase is indicated by the open arrowheads. (D) The ratios of the signal intensities of Hsp70 from lanes 1 and 2.

tyrosinase activity was demonstrated from the data for each harvested cell line and were greatest in the MNT-1 cells, lower in the HM3KO cells and lowest in the G-361 cells (Fig. 1B and $\mathrm{C}$ ). Western blot analysis revealed that the molecular
A

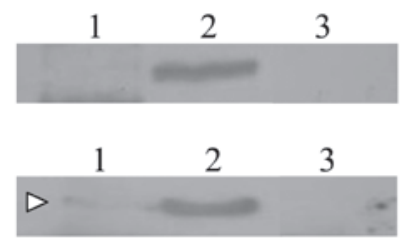

C

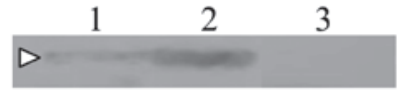

D

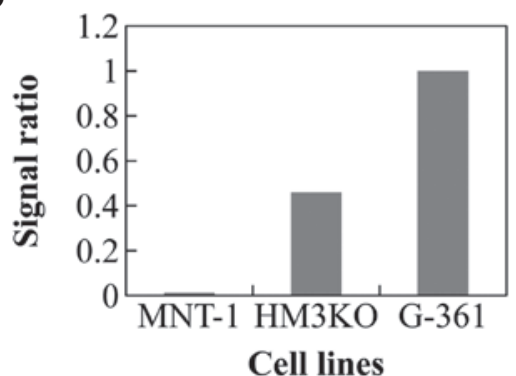

Figure 4. Immunoprecipitation of Hsp70 with anti-tyrosinase in HM3KO and G-361 cells. Extracts were prepared from (A) MNT-1, (B) HM3KO and (C) G-361 cells. Each extract was immunoprecipitated with anti-tyrosinase antibody. The immunoprecipitated products and extracts were analyzed by western blot analysis with anti-Hsp70 antibody. Lane 1, immunoprecipitated fraction with anti-tyrosinase; lane 2, extract without the immunoprecipitation treatment; lane 3, nonimmune IgG. The immuoprecipitated Hsp70 with antityrosinase is indicated by the open arrowheads. (D) The ratios of the signal intensities of $\mathrm{Hsp} 70$ from lanes 1 and 2.

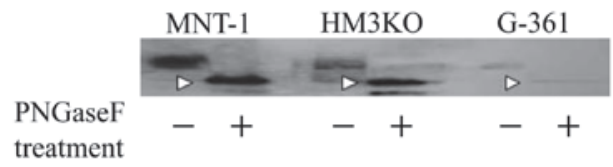

Figure 5. PNGase $\mathrm{F}$ treatment of the tyrosinase from each cultured melanoma cell line. Each $15-\mu \mathrm{g}$ extract was treated with PNGase F and analyzed by western blot analysis with anti-tyrosinase antibody. The signals from the PNGase F-treated tyrosinase are indicated by open arrowheads.

masses of HM3KO- and G-361-derived tyrosinase were slightly lower than that derived from MNT-1 cells, and the production level of tyrosinase was greatest in the MNT-1 cells, lower in the HM3KO cells and lowest in the G-361 cells (Fig. 1D).

Treatment of melanoma cell lines by glycosylation inhibitors, DNJ and DMJ. A melanoma cell line, MNT-1, was treated with DNJ and DMJ, which are protein glycosylation inhibitors. Western blot analysis using tyrosinase antibodies demonstrated differences in the molecular masses of the tyrosinase treated with each inhibitor (Fig. 2A). The molecular mass of the tyrosinase treated with DMJ was approximately that of the HM3KO and G-361 cells. The melanin production and tyrosinase activity of those cells significantly decreased following the treatments, and were lower than those of untreated MNT-1 cells (Fig. 3B and C).

Immunoprecipitation of Hsp70 with tyrosinase antibodies. The crude extracts of MNT-1 treated with or without the glyco- 
A

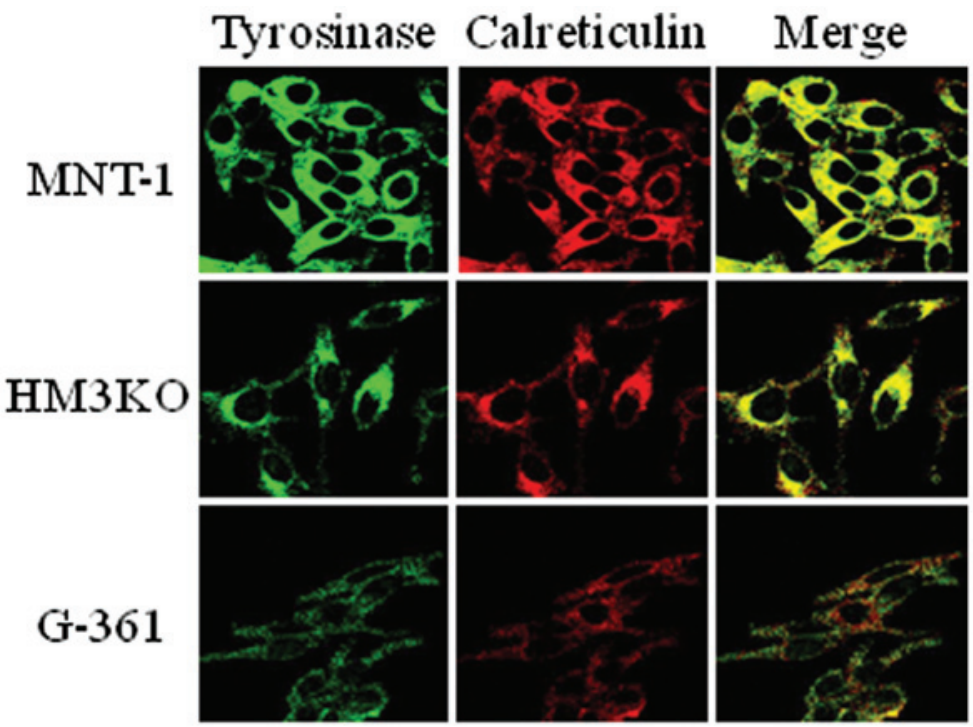

B

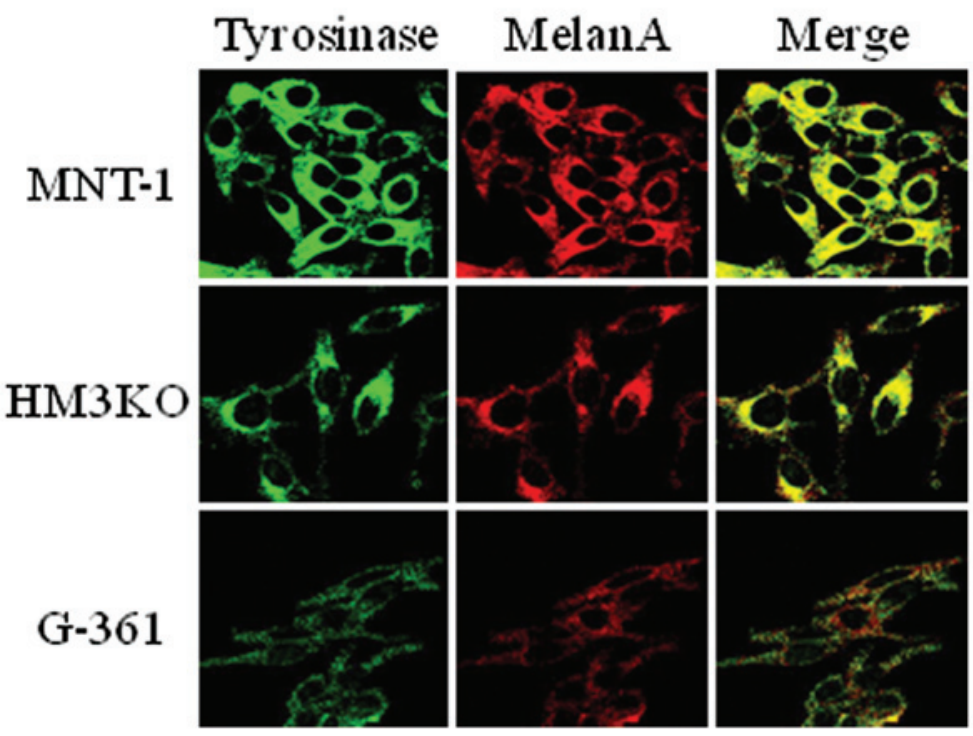

Figure 6. Subcellular localization of tyrosinase in each cultured melanoma cell line, MNT-1, HM3KO and G-361. The subcellular localization of tyrosinase was observed by laser scanning confocal microscopy (magnification, x40; FV1000IX81S-HU; Olympus Co., Tokyo, Japan). Calreticulin and melan-A were used as marker proteins for endoplasmic reticulum (ER) and melanosomes, respectively.

sylated inhibitors were immunoprecipitated with tyrosinase antibody and subjected to western blot analysis with Hsp70 antibody (Fig. 3). In the untreated MNT-1 cells, the reaction signal was almost unidentifiable (Fig. 3A and D). Conversely, the same fractions in DNJ- and DMJ-treated MNT-1 cells were indicated to be present in the immunoprecipitated complex of Hsp70 and tyrosinase (Fig. 3B-D). These data suggested that the misfolded tyrosinase resulting from the immature glycosylation in DNJ- and DMJ-treated MNT-1 cells was transported for other processes via Hsp70 and was not transported to the melanosomes for melanin production.

The Hsp70 immunoprecipitation in the extracts of HM3KO and G-361 cells were examined using the tyrosinase antibody. Although the reaction product was not recognized in MNT-1 cells, the presence of the immunoprecipitated complex of Hsp70 and tyrosinase was detected in the HM3KO and G-361 cells (Fig. 4B-D). The intensity of the interaction was greater than that observed in the inhibitor-treated MNT-1 cells (Fig. 3D and 4D).

\section{Discussion}

In the present study, three types of cultured MM cell lines (MNT-1, HM3KO and G-361) were examined. It was demonstrated that their melanin production levels differed from each other, and a positive correlation between the melanin production and tyrosinase activity was observed (Fig. 1A-C). The quantities of tyrosinase were correlated with the melanin production and the tyrosinase activity (Fig. 1D). The differences among the molecular masses of tyrosinases (Fig. 1D) almost disappeared as a result of digestion with PNGase F (Fig. 5). In addition, there were no significant differences in the mRNA levels of tyrosinase among these cell lines (data not shown) and, in each cell line, tyrosinase was present at the ER 
and in the melanosome (Fig. 6). These results suggested that the differences in the melanin production and the tyrosinase activity among these cell lines were due to the glycosylation of tyrosinase at a post-translational level. However, the nucleotide sequence of cDNA encoding tyrosinase from G-361 cells (accession no. AB775901) had a nucleotide substitution that was demonstrated to have a critical affect on pigmentation (15). No mutations were observed to be significantly correlated with melanin production and tyrosinase activity in the nucleotide sequences of cDNA encoding tyrosinase from MNT-1 (AB775899) and HM3KO (AB775900) cells. It is possible that the differences measured in the melanin production and the tyrosinase activity between MNT-1 and HM3KO cells were mainly due to disorders in the glycosylation process of tyrosinase. Although the decreases in melanin production and tyrosinase activity in G-361 cells were caused by a mutation in its nucleotide sequence, the decrease of the molecular mass of tyrosinase may have been due to an abnormal glycosylation at a post-transcriptional level.

Therefore, we evaluated the influence of the abnormal glycosylation in tyrosinase production on the activity and melanin production of MNT-1 cells using two glycosylation inhibitors, DNJ and DMJ. The tyrosinases treated with each inhibitor demonstrated differences in molecular mass. Furthermore, melanin production and tyrosinase activity decreased due to DNJ and DMJ treatment. These data suggested that it is difficult for MNT-1 to synthesize melanin with immature glycosylated tyrosinase. The endogenous chaperone proteins and lectin-like proteins were demonstrated to be important in maintaining accurate protein maturation and degrading misfolded protein by translocation into proteolytic pathways, such as the ubiquitin/proteasome system and the ER-associated protein degradation pathway $(16,17)$. Therefore, in this study, it was further investigated whether the chaperone protein was associated with the immature glycosylated tyrosinase, using an immunoprecipitation assay. The extracts of cells treated with each glycosylation inhibitor were immunoprecipitated with anti-tyrosinase antibody and analyzed by western blot analysis with anti-Hsp70 antibody (Fig. 4). In the non-treated MNT-1 cells, the reaction signal was almost undetectable; however, the same fractions in DNJ- and DMJ-treated MNT-1 cells were present in the immunoprecipitated complex of $\mathrm{Hsp} 70$ and tyrosinase (Fig. 3). These data indicated that the immature glycosylated tyrosinase reaction with the chaperone protein, HSP70, and may have been transported into the proteolytic pathways to maintain protein quality. In addition, each extract from the HM3KO and G-361 cells was immunoprecipitated with anti-tyrosinase antibody, and the reactions with anti-HSP70 antibody were also confirmed in the HM3KO and G-361 cells (Fig. 4). These tyrosinases associated with Hsp70 were translocated to the proteolytic pathways, leading to decreased activity and melanin production.

In this study, it was demonstrated that the glycosylation of tyrosinase is a determinant of melanin production in at least three types of cultured melanoma cells. Tyrosinase expressed in MNT-1 cells exhibited accurate glycosylation, and demonstrated enzyme activity and melanin production. HM3KO and G-361 cells also expressed tyrosinase; however, the tyrosinase was not well maintained in the active form.

\section{Acknowledgements}

This study was supported in part by a grant from Hirosaki University (Hirosaki, Japan) for the promotion of International Scientific Research.

\section{References}

1. Ando H, Kondoh H, Ichihashi M and Hearing VJ: Approaches to identify inhibitors of melanin biosynthesis via the quality control of tyrosinase. J Invest Dermatol 127: 751-761, 2007.

2. Wang $\mathrm{N}$ and Hebert DN: Tyrosinase maturation through the mammalian secretory pathway: bringing color to life. Pigment Cell Res 19: 3-18, 2006.

3. Uong A and Zon LI: Melanocytes in development and cancer. J Cell Physiol 222: 38-41, 2010.

4. Kushimoto T, Basrur V, Valencia J, Matsunaga J, Vieira WD, Ferrans VJ, Muller J, Appella E and Hearing VJ: A model for melanosome biogenesis based on the purification and analysis of early melanosomes. Proc Natl Acad Sci USA 98: 10698-10703, 2001.

5. Watabe H, Valencia JC, Le Pape E, Yamaguchi Y, Nakamura M, Rouzaud F, Hoashi T, Kawa Y, Mizoguchi M and Hearing VJ: Involvement of dynein and spectrin with early melanosome transport and melanosomal protein trafficking. J Invest Dermatol 128: 162-174, 2008.

6. Godbole D, Mojamdar M and Pal JK: Increased level of p27 subunit of proteasomes and its co-localization with tyrosinase in amelanotic melanoma cells indicate its direct role in the regulation of melanin biosynthesis. Cell Biol Int 30: 895-902, 2006.

7. Ohashi A, Funasaka Y, Ueda M and Ichihashi M: c-KIT receptor expression in cutaneous malignant melanoma and benign melanotic naevi. Melanoma Res 6: 25-30, 1996.

8. Ohshima Y, Yajima I, Takeda K, Iida M, Kumasaka M, Matsumoto $Y$ and Kato M: c-RET molecule in malignant melanoma from oncogenic RET-carrying transgenic mice and human cell lines. PLoS One 5: e10279, 2010.

9. Peebles PT, Trisch T and Papageorge AG: Isolation of four unusual pediatric solid tumor cell lines. Pediat Res 12: 485, 1978.

10. Katagata Y and Hirayama T: Unexpected expression of Hsp47, a replacement of one amino acid ( Val $7 \mathrm{Leu})$ in the amino terminal region, in cultured human tumorigenic cell lines. J Dermatol Sci 49: 33-38, 2008.

11. Ando H, Funasaka Y, Oka M, Ohashi A, Furumura M, Matsunaga J, Matsunaga N, Hearing VJ and Ichihashi M: Possible involvement of proteolytic degradation of tyrosinase in the regulatory effect of fatty acids on melanogenesis. J Lipid Res 40: 1312-1316, 1999.

12. Branza-Nichita N, Negroiu G, Petrescu AJ, Garman EF, Platt FM, Wormald MR, Dwek RA and Petrescu SM: Mutations at critical $\mathrm{N}$-glycosylation sites reduce tyrosinase activity by altering folding and quality control. J Biol Chem 275: 8169-8175, 2000.

13. Asryants RA, Duszenkova IV and Nagradova NK: Determination of Sepharose-bound protein with Coomassie brilliant blue G-250. Anal Biochem 151: 571-574, 1985.

14. Choi H, Ahn S, Chang H, Cho NS, Joo K, Lee BG, Chang I and Hwang JS: Influence of N-glycan processing disruption on tyrosinase and melanin synthesis in HM3KO melanoma cells. Exp Dermatol 16: 110-117, 2007.

15. Ibarrola-Villava M, Hu HH, Guedj M, Fernandez LP, Descamps V, Basset-Seguin N, Bagot M, Benssussan A, Saiag P, Fargnoli MC, et al: MC1R, SLC45A2 and TYR genetic variants involved in melanoma susceptibility in southern European populations: results from a meta-analysis. Eur J Cancer 48: 2183-2191, 2012.

16. Chien V, Aitken JF, Zhang S, Buchanan CM, Hickey A, Brittain T, Cooper GJ and Loomes KM: The chaperone proteins HSP70, HSP40/DnaJ and GRP78/BiP suppress misfolding and formation of $\beta$-sheet-containing aggregates by human amylin: a potential role for defective chaperone biology in Type 2 diabetes. Biochem J 432: 113-121, 2010.

17. Mikami K, Yamaguchi D, Tateno H, Hu D, Qin SY, Kawasaki N, Yamada M, Matsumoto N, Hirabayashi J, Ito Y and Yamamoto K: The sugar-binding ability of human OS-9 and its involvement in ER-associated degradation. Glycobiology 20: 310-321, 2010. 